\title{
Magnetic Resonance Imaging Image Segmentation and Brain Tumour Detection Using Pulse-Coupled Neural Networks
}

\author{
Louiza Dehyadegari $^{1^{*}}$ and Somayeh Khajehasani \\ ${ }^{1}$ Department of Electronic Science, Sirjan University of Technology, Sirjan, Iran \\ ${ }^{2}$ Department of Computer Science, Sirjan University of Technology, Sirjan, Iran \\ *Corresponding author: dehyadegari@sirjantech.ac.ir
}

Published online: 31 May 2021

To cite this article: Louiza Dehyadegari and Somayeh Khajehasani (2021). Magnetic resonance imaging image segmentation and brain tumour detection using pulse-coupled neural networks. Journal of Engineering Science, 17(1), 1-16, https://doi.org/10.21315/ jes2021.17.1.1.

To link to this article: https://doi.org/10.21315/jes2021.17.1.1

\begin{abstract}
Image processing can be defined as a functional structure to correct and change the images viewed and their interpretation. One of the applications of digital image processing is using image processing techniques in the component and image segmentation. One of these techniques is magnetic resonance imaging (MRI) in the medical world. In this article, a brain tumour detection system and various anomalies and abnormalities are presented where image pre-processing and preparation include image enhancement, filtering and noise reduction. Then image segmentation is done by a pulse neural network. Next, the image features are extracted and finally, the tumour and abnormal area are separated from the normal area by the algorithms. In this research, the feature selection and integration method are used and the most important statistical features of brain MRI images are used to improve brain tumour detection. Along with the studies done and the implementation of tumour detection systems, the following suggestions can be provided for future researches and the tumour detection system will work more efficiently. The pulsecoupled neural network (PCNN) can be used for image segmentation in the pre-processing stage, especially in the image filtering.
\end{abstract}

Keywords: image processing, segmentation, pulse-coupled neural network, feature selection, image filtering

\section{INTRODUCTION}

With the dramatic increase in the volume of information and advances in medicine, there is a growing need for methods and techniques that can provide data efficiently and extract information. The tumour detection by data mining techniques has led to the link between the medical world and these techniques. 
The correct diagnosis of these tumours is essential to predict tumour growth. ${ }^{1}$ The new technology can detect, diagnose and evaluate common and rare diseases through non-invasive imaging. Doctors try to detect anomalies and abnormalities by these techniques and magnetic resonance imaging (MRI) segmentation. Nowadays, MRI is widely used for the diagnosis of brain tumours. ${ }^{2}$ Segmentation of brain tumours and surrounding abnormal tissues based on MRI images can provide doctors with a direct understanding of tumours and assistance for analysis and treatment. Therefore, brain tumour segmentation is regarded as an essential step in MRI analysis. ${ }^{3}$ At present, there is no standard segmentation technique that can provide satisfactory results for all imaging applications such as brain MRI, brain tumour detection, etc.

The optimal selection of features, tissues and brain and non-brain elements is crucial for the segmentation of brain images. ${ }^{2-8}$ Some methods such as deep neural networks, ${ }^{6}$ convolutional neural networks, ${ }^{9}$ interactive Bayesian multivariate deep neural network (IBMDNN) classifier, ${ }^{10}$ deep convolutional neural networks, ${ }^{4}$ cascading handcrafted features and convolutional neural network ${ }^{5}$ have been used for brain tumour segmentation and classification. In this article, we have tried to use the important and essential feature selection techniques to distinguish normal images from abnormal images in the best way with the least error. Pulse-coupled neural network (PCNN), known as the third generation of the neural network model and one of the newest bio- algorithms and models, is the key issue of this article. PCNN is a biological model based on the mammalian visual nucleus proposed by Eckhorn et al. ${ }^{11}$ The proposed model can be used as an analysis and evaluation software for brain tumour detection. The purpose of this project is to process MRI images to diagnose brain tumours. Thus image processing methods are used to extract data and then these data are examined and classified using PCNN. In the second part, the concepts related to the processing of MRI images and the challenges on these images and the PCNN model are discussed. The methodology used in this research is discussed in detail in Section 3.

\section{THEORY AND PRINCIPLES OF RESEARCH}

Data mining is a growing field of study tied to other fields such as artificial intelligence, statistics, vision, parallel computing and image processing. Data mining techniques such as decision tree, ${ }^{1}$ support vector machine (SVM), ${ }^{12}$ artificial neural networks like multilayer perception (MLP) model ${ }^{13}$ and fuzzy C-means algorithm, ${ }^{14}$ are beneficial for finding medical decisions to detect brain tumour and cancer diseases. Among the advances in science and technology due to the high computational efficiency, medical imaging is the most prominent because it is desirable to show the human body's internal structures. 
Brain tumour detection is a very sensitive and complex matter. Therefore, accuracy and reliability are of particular importance. The correct detection of these tumours is necessary to predict tumour growth. One of the imaging techniques is MRI. ${ }^{15}$ MRI allows researchers to study the brain's functional and physical features with the lowest risk for the patient. It is up to the doctors to test the images and identify the region of interest (ROI) to diagnose. ROI is then segmented into anatomical structures and their possible constituent abnormalities. This anatomical segmentation is similar to the computerised segmentation of images. MRI, using magnetism and radio frequency (RF), produces cross-sectional diagnostic images of the body. ${ }^{16}$ The magnetic resonance image segmentation is an important step for many applications, including identifying anatomical ROIs for diagnosis, treatment, surgical planning, pre-processing for multidimensional image recording and tumour volume measurement. ${ }^{17}$ The brain tissue segmentation, including three parts of grey matter (GM), white matter (WM) and cerebrospinal fluid region (CSF) of the brain resonance images, plays an important role in clinical issues and neuron science studies. MRI brain tumour segmentation is difficult due to the following factors:

1. Deformation of a non-tumour structure due to the tumour mass effect.

2. Brain tissue infiltration and oedema (swelling). Swelling appears around the tumour and appears mainly white matter.

3. The gradual transition from tumour to swelling often makes it difficult to detect these two structures' border.

In image segmentation, the pixels are segmented in the regions and thus the object areas are defined. The common MRI segmentation approaches include threshold, edge detection, clustering, genetic algorithms, neural networks and probabilities.

Medical image segmentation is an unresolved issue. The methods such as neural networks and statistical methods are applied for this purpose. However, the image and texture properties prevent the production of reliable results. PCNN is a neural network model that includes parallel processing, which can solve computational complexity problems. This neural network model can be used in target detection, segmentation, edge extraction and object identification. The use of PCNN for objects was introduced by Johnson and was developed by Kinser and his colleagues. ${ }^{18}$ PCNN significant advantage is the invariance of the generated time signal for image rotation, dilation, or reversal. ${ }^{19,20}$ 
The PCNN model is a biological model based on the mammalian visual nucleus proposed by Eckhorn et al. ${ }^{11}$ In biology, the combined connections between cells are observed. Therefore, the PCNN model is used because it simulates intra-neuron connections. PCNN is a two-dimensional (2D) singlelayer neural network that is a pulse-coupled neural network connected with image pixels. Each pixel is related to one neuron.

For image processing, $N \times N$ 2D image matrix is represented as an $N \times N$ array of PCNN neuron connections. Therefore, we can say that a neuron matches an image pixel and the pixel intensity will be the trigger value. The pixel neighbourhood will be according to the neuron neighbourhood. In common neural networks, each neuron has several inputs and outputs.

In the proposed technique, the PCNN algorithm is used to align the two-dimensional brain images and tumour detection and PCNN is used for classification. In this method, the input images are first prepared and cleaned up by pre-processing algorithms for the next step. The efficient and important features come out of the image by a variety of feature extraction methods. Then different regions of the brain are segmented by PCNN and finally, the tumour position is identified. A significant advantage of $\mathrm{PCNN}$ is the invariance of the generated time signal for the image rotation, dilation, or reversal. The advantage of the PCNN model over common neural network models is that digital image segmentation does not require sample preparation. However, the threshold parameters, coupling coefficient, weighting factors and other parameters must be adjusted. The segmentation quality is affected during loop repeats.

\section{THE PROPOSED ALGORITHM}

The research framework of this article can be summarised in a diagram as follows. Each of these implemented steps will be described separately and the output of each step will be specified individually. 


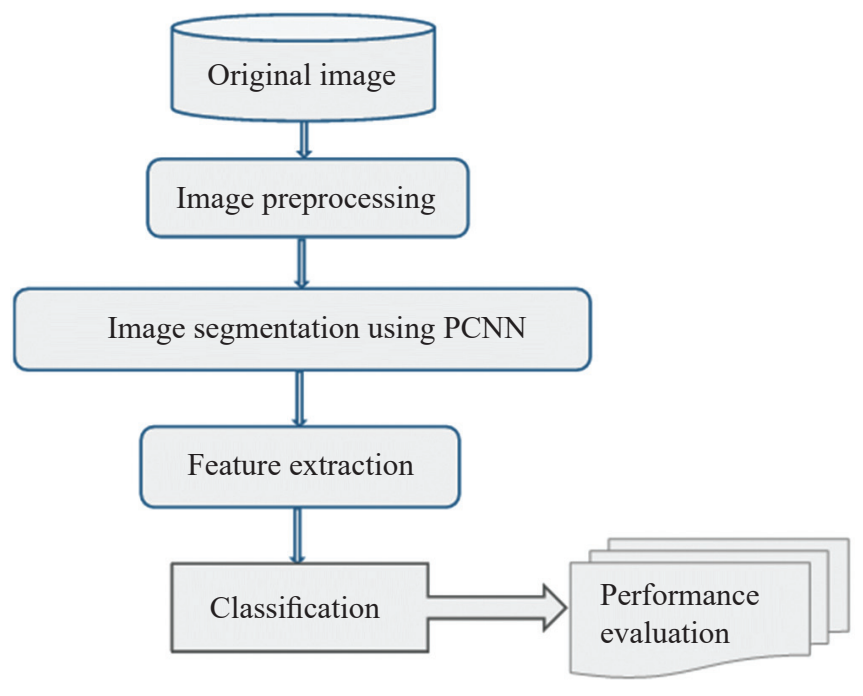

Figure 1: The proposed algorithm diagram.

The proposed algorithm (as shown in Figure 1) is based on the brain MRI images, including 14 normal and 87 abnormal (benign and malignant tumours) images from a real human dataset. This dataset is used to evaluate the efficiency and includes $256 \times 256$ pixels brain images. The images are collected from the Harvard Medical School website. Figure 2 shows some examples of normal and abnormal images from this dataset.

\subsection{Image Pre-Processing}

Before segmentation and after receiving the dataset, image pre-processing is required. It is necessary to filter and remove high-frequency noises to make the image smoother and more uniform to detect the image changes. ${ }^{22}$ Histogram equalisation is a method in image processing of contrast adjustment using the image's histogram and is the best image enhancement method. ${ }^{23}$ The median filter tends to maintain the resolution of edges while removing the noise. A $4 \times 4$ window is used to do this. In a median filter with size $N \times N$, if $N$ is even, the average of the four central elements defined as the middle pixel. At every window slide, four pixels are updated at the same time. Numerical values of image pixels are averaged at any time for the central pixel. Figure 3 shows the main image and the image after applying the median filter and histogram equalisation in the proposed method. 


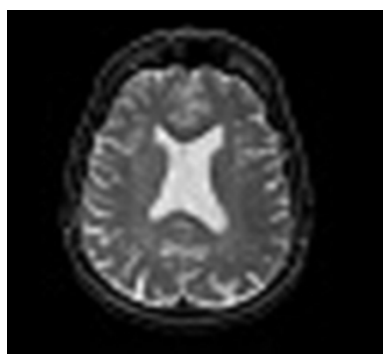

(a)

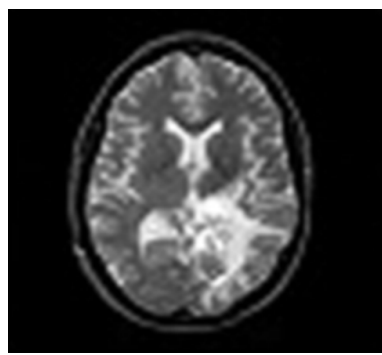

(d)

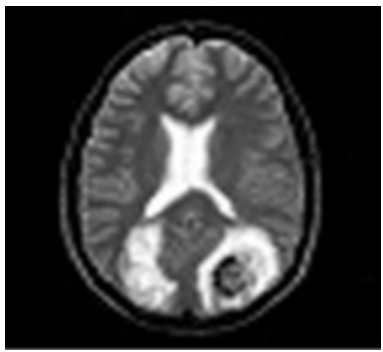

(g)

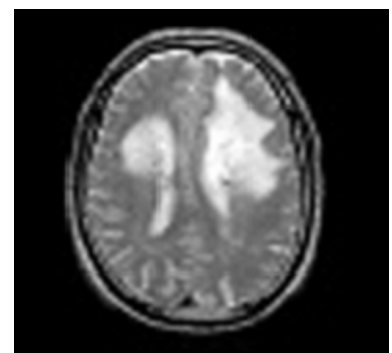

(b)

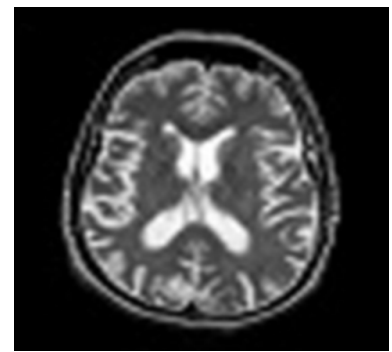

(e)

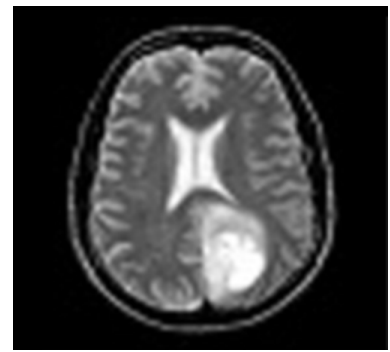

(h)



(c)



(f)

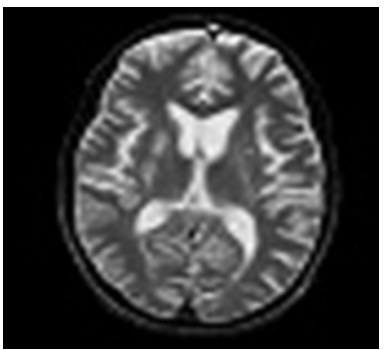

(i)

Source: Harvard Medical School ${ }^{21}$

Figure 2: Different types of normal and abnormal images: (a) Normal, (b) Meningioma, (c) Metastatic bronchogenic carcinoma, (d) Glioblastoma, (e) Alzheimer's disease, (f) Alzheimer's disease with visual agnosia, (g) Sarcoma, (h) Glioma and (i) Pick's disease. 


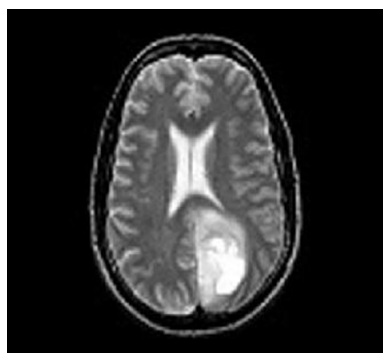

(a)

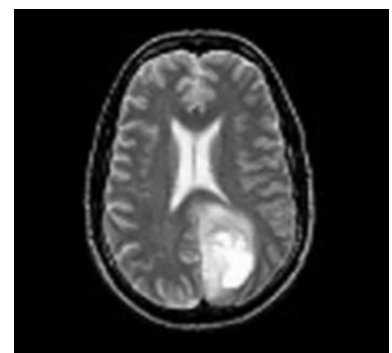

(b)

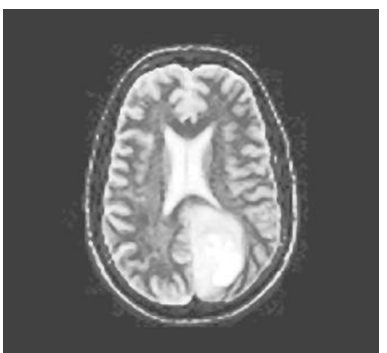

(c)

Figure 3: MRI image: (a) Before pre-processing, (b) After applying the median filter and (c) After histogram equalisation

\subsection{Segmentation by Feedback PCNN (FPCNN) Model}

Segmentation is a technique that groups pixels in different regions. FPCNN ${ }^{18}$ sends the output information back to the input in a return mode. ${ }^{24}$ In this method, by giving feedback in the input, we can obtain more logical results. It segments ROIs by consecutive repetitions. The PCNN model's success mainly depends on network parameters' adjustment, including the link (connection) parameter, the threshold value and $\mathrm{M}$ and $\mathrm{W}$ matrices that are synaptic weight matrices. Figure 4 shows the structure of PCNN and Equations 1 and 2 show the relationship between inputs and outputs in PCNN.

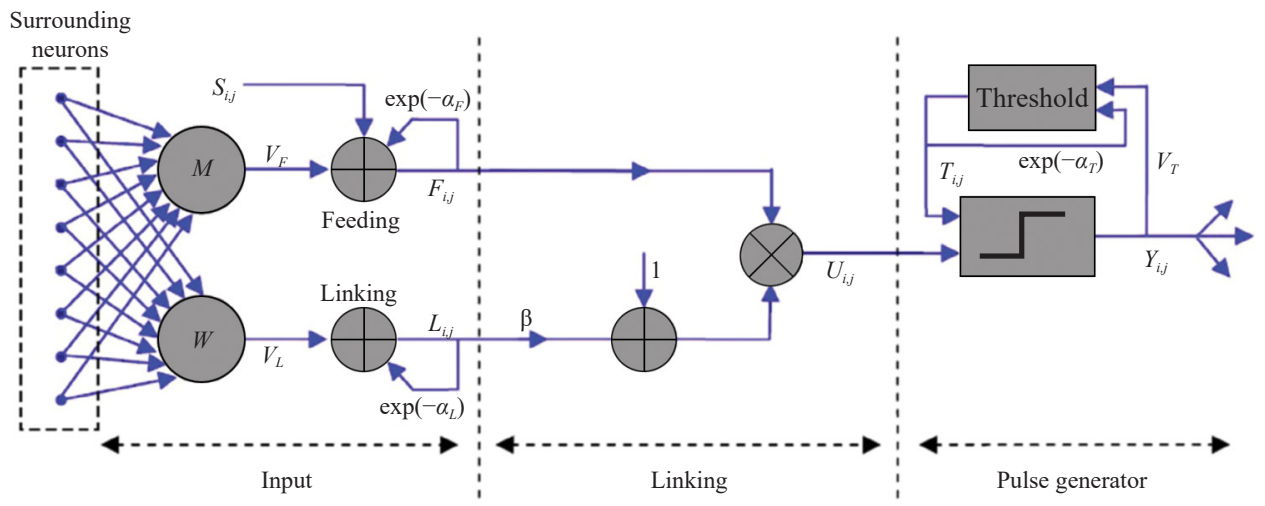

Figure 4: The structure of PCNN. 


$$
\begin{aligned}
& F_{i j}[n]=e^{-\alpha_{F}} F_{i j}[n-1]+V_{F} \sum_{k, l} m_{i, j, k, l} Y_{i j}[n-1]+S_{i j} \\
& L_{i j}[n]=e^{-\alpha_{L}} L_{i j}[n-1]+V_{L} \sum_{k, l} w_{i, j, k, l} Y_{i j}[n-1]
\end{aligned}
$$

In which $F_{i j}[n]$ is the preliminary input of neuron, $L_{i j}[n]$ is the linking output, $S_{i j}[n]$ is the input drives for image pixels in location $(i, j), Y_{i j}[n]$ is the outputs of neurons, $\beta$ is the linking coefficient, $M_{i j k l}$ and $W_{i j k l}$ are constant Gaussian weight functions with distance criterion, $V_{F}$ and $V_{L}$ are the main weights and $k, l$ are the Gaussian function parameters.

The main difference between PCNN and FPCNN is updating the input pixels by feedback from the output. Figure 5 shows the structure of FPCNN and Equations 3 and 4 show the relationship between inputs and outputs in FPCNN.

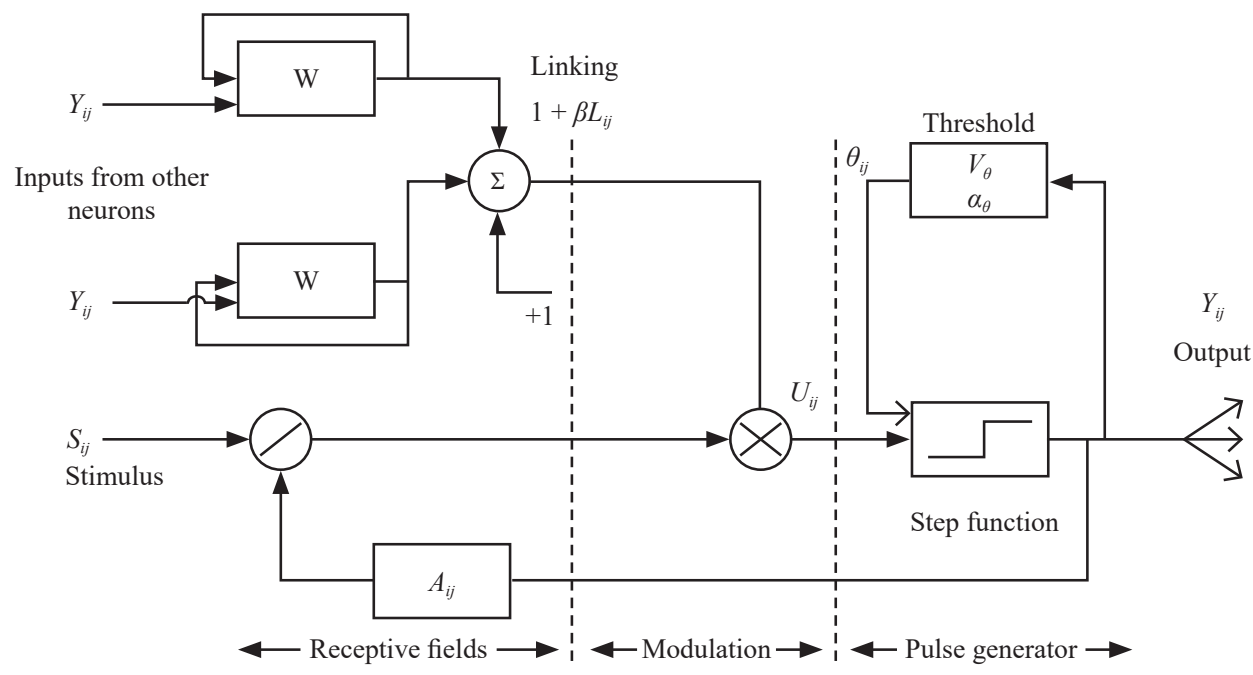

Figure 5: The structure of FPCNN.

$$
\begin{aligned}
& A_{i j}[n]=\exp \left(-\alpha_{A}\right) A_{i j}[n-1]+V_{A} Y_{i j}[n] \\
& A_{i j}[n]=\frac{S_{i j}[n-1]}{A_{i j}[n-1]}
\end{aligned}
$$

In which $\alpha_{A}$ is a reduction constant that affects the previous memory of $A_{i j}$ and $V_{A}$ is regulation constant. 
The coupling mechanism forms the core of the PCNN theory. Coupling is the connection between neurons. While using the PCNN model for image segmentation, the coupling between neurons allow neurons with similar brightness and are adjacent to each other to pulse simultaneously. By pulse expansion, the image parts are segmented. The coupling part of neurons has two parameters: $W_{i j k l}$ and $\beta . W_{i j k l}$ represent the degree $\left(M_{i j k l}\right.$ and $W_{i j k l}$ : constant Gaussian functions with distance criterion) that the central neuron is affected by its peripheral neurons. This parameter determines the coupling power between neurons that shows the information transmission power from the neighbouring neurons to the central neuron. $\beta$ (linking factor) indicates the power between the peripheral neurons' synapses and the central neuron and reflects the difference between the central neuron and neighbouring neurons. Its higher amount leads to easier stimulation of the central neuron. In image segmentation, PCNN neurons receive the feed input from the image pixels and the neighbouring neurons' connection inputs. Its graphical representation is shown in Figure 6. In which $X$ is the input MRI for PCNN, $Y$ is the segmentation output of PCNN, $F$ is the activation function of a neuron and $L$ verified the neurons' neighbourhood.

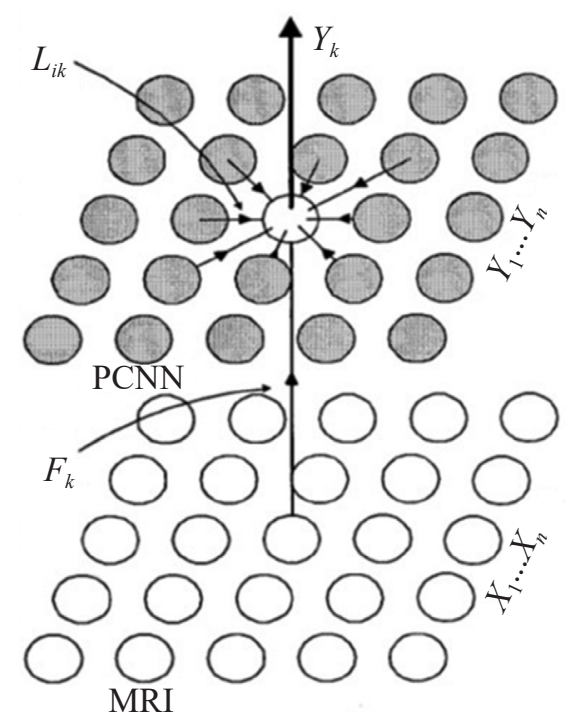

Figure 6: Neuron connection and feed relations. ${ }^{25}$

The PCNN segmentation algorithm is run as follows: Since all neurons fire at $t=0$, the segmentation starts at $t=1$. All $F_{k} \mathrm{~s}$ are normalised with the maximum intensity so that the neurons related to the brightest pixel fire at $t=1$. When a neuron fires, all the $L_{K}$ connection field neurons receive a pulse in the fired neuron neighbourhood. If a sufficient connection is obtained, $T_{k}<U_{k}$, these neurons are 
also fired where $T_{k}$ is the distance between neuron $k$ and its neighbourhoods. $U_{k}$ is the threshold of neighbourhood distance for firing the neighbourhood neurons. Therefore, neurons may be evaluated several times before firing or may not be fired over a period of time. As mentioned before, the FPCNN model is the same as the PCNN model, except that the output information returns to the input. Thus, all of the above is true for the FPCNN model. Table 1 shows the values of the FPCNN parameters used in the implementations. These values are cited from reference. ${ }^{26}$

Table 1: Values of the FPCNN network parameters.

\begin{tabular}{crl}
\hline Constant & Value & Concept \\
\hline$\beta$ & 0.02 & Linking factor \\
$V F$ & 0.01 & Feed connection \\
$V L$ & 2.00 & Link connection \\
$V \theta$ & 3.00 & Threshold value \\
VA & 1.00 & Weighted time mean connection \\
$\alpha F$ & 30.00 & Feed weakening \\
$\alpha L$ & 0.15 & Link weakening \\
$\alpha \theta$ & 0.06 & Threshold weakening \\
$A \alpha$ & 0.06 & Weighted time mean weakening \\
\hline
\end{tabular}

After applying this step, the ROI are obtained and it results in the segmentation of the main image and the identification of the important features. The results obtained by implementing FPCNN for a number of brain images are shown in Figure 7.

\subsection{Feature Extraction}

According to the processed and segmented images in the previous steps, a set of features is selected from each image. Before the classification model is developed, the meaningful features of ROIs generated in the segmentation need to be extracted and used as the classification model's input. Mean, standard deviation, skewness, kurtosis, energy and entropy statistical properties are used to extract the feature. They are calculated using the distribution probability of the intensity levels between $H_{D C}, H_{A C 1}, H_{A C 2}$ and $H_{A C 3}$ histograms. The binary image obtained from the FPCNN model is divided into $8 \times 8$ blocks that have no overlap. All of these blocks are then converted to Domain Cosine Transformation (DCT) blocks in a frequency range. The first coefficient of DCT in a DCT block is called the DC coefficient, which represents the average values of a block's intensity. The other coefficients of DCT blocks are called AC coefficients, which correspond to different frequencies. 
After DCT conversion, all blocks' DC coefficients and the three initial AC coefficients (AC1, AC2 and AC3) are selected in a zigzag pattern. All of these $\mathrm{DC}$ and $\mathrm{AC}$ coefficients are chosen to construct the histograms.
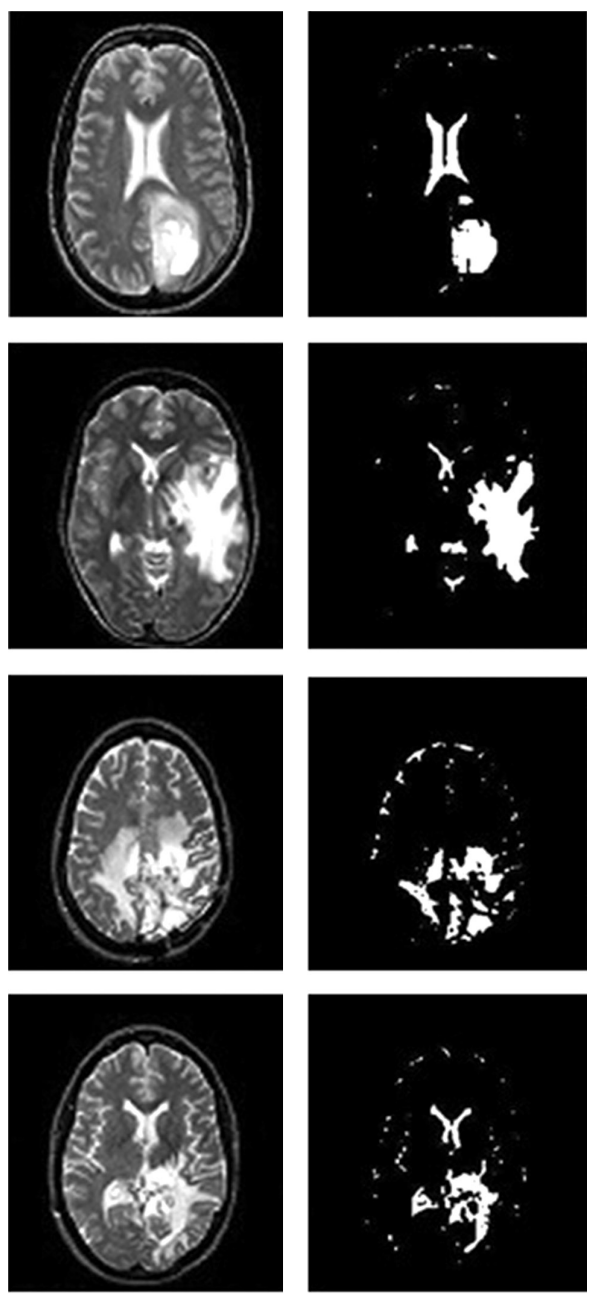

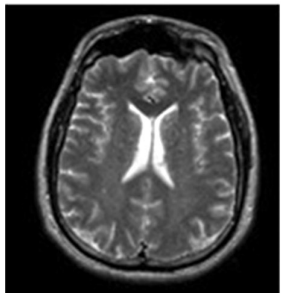

(a)



(b)

Figure 7: (a) Main image and (b) Segmented image. 


\subsection{Classification and Performance Evaluation}

The proposed technique in El-Dahshan et al. ${ }^{26}$ based on PCNN for segmentation, discrete wavelet transform (DWT) for feature extraction phase, has used principal component analysis (PCA) algorithm to reduce the dimension and finally the classification is done on the data. In this article, 101 images, including 14 normal data and 87 abnormal data, are used.

Fawcett ${ }^{27}$ in 2006 used two DWT and SVM techniques. In Powers, ${ }^{28}$ the combination method of PCA and SVM is used. By this method, PCA is used to reduce the dimension and eventually, SVM is applied to classify the normal and abnormal images. Also, in Dubey, Hanmandlu and Gupta, ${ }^{29}$ the first DWT is used for feature extraction, PCA is applied for dimension reduction and then the $k$-nearest neighbour (KNN) method is used for classification. These algorithms are used as the base methods to compare with the proposed method and the results are presented in Tables 2 to 4 .

Table 2: The error percentage obtained in the proposed methods of SVM, KNN and MLP.

\begin{tabular}{cc}
\hline Error rate (percentage) & Classifier \\
\hline 2.97 & SVM \\
1.98 & KNN \\
1.98 & MLP \\
\hline
\end{tabular}

Table 3: Sensitivity, FN, FP, TN, FN and specificity obtained in the proposed and previous ${ }^{26}$ methods.

\begin{tabular}{lccccccc}
\hline & & TP & TN & FP & FN & $\begin{array}{c}\text { Sensitivity } \\
(\%)\end{array}$ & $\begin{array}{c}\text { Specificity } \\
(\%)\end{array}$ \\
\hline Proposed method & SVM & 84 & 14 & - & 3 & 96.5 & 100.0 \\
& KNN & 85 & 14 & - & 2 & 97.7 & 100.0 \\
& MLP & 85 & 14 & - & 2 & 97.7 & 100.0 \\
PCNN + DWT + PCA & & 87 & 13 & 1 & - & 100.0 & 92.8 \\
\hline
\end{tabular}

We could change the problem into a classification problem with two normal and abnormal classes due to two different normal and abnormal types. We consider the degree of efficiency assessment as the number of correct classification or classification accuracy. The classification is a technique for classifying input patterns in comparable classes. There are many classification algorithms for classifying brain MRI and they have different strengths and weaknesses. Classification will be a two-class problem, regarding that images are 
converted into two classes of normal and abnormal or tumour and non-tumour. MLP neural network and KNN and SVM algorithms are used for classification. The classification accuracy, sensitivity, specificity criteria and error rate will be explained. ${ }^{26,27}$

Table 4: The comparison of sensitivity, specificity and accuracy of some methods with the proposed method.

\begin{tabular}{|c|c|c|c|}
\hline & Specificity (\%) & Sensitivity (\%) & Accuracy $(\%)$ \\
\hline The proposed method & 98.02 & 100.00 & 97.50 \\
\hline PCNN + DWT + PCA method ${ }^{26}$ & 99.00 & 92.80 & 100.00 \\
\hline DWT + SVM method ${ }^{29}$ & 96.00 & 96.00 & 95.50 \\
\hline PCA + SVM method ${ }^{30}$ & 85.00 & 84.00 & 89.00 \\
\hline DWT + PCA + KNN method ${ }^{31}$ & 98.00 & 97.00 & 96.00 \\
\hline
\end{tabular}

As it is evident in Table 3, FP is zero in the proposed method, which indicates that none of the proposed methods wrongly classify the normal image as part of abnormal images. On the other hand, Table 4 compares the sensitivity, specificity and accuracy of the proposed method with some other methods. As shown in the table, the proposed method can perform better than most other methods and classify normal and abnormal images more successfully.

\section{CONCLUSION AND SUGGESTIONS}

Today, tumour detection systems are still emerging and a lot of work has to be done. A large amount of information in brain MRI images has caused no standard segmentation technique that can have satisfactory results for all imaging applications such as brain MRI, brain cancer diagnosis, etc. Thus in this regard, there is a lot of work to do to improve the diagnosis. The optimal selection of brain and non-brain features, tissues and elements is crucial for brain image segmentation. Therefore, in this article, we try to use the important techniques of feature selection. Unlike previous studies that used the input image as the PCNN input, we applied the statistical properties obtained at an earlier step as the network input for segmentation.

This study's importance can be examined from different perspectives: brain tumour detection and the diagnosis of cancer and other types of abnormalities. We are providing a highly accurate method to identify diseases and abnormalities. Predicting various diseases and finding types of tumours and abnormalities, if any, in different forms and sizes. 
In this article, brain MRI images' most important statistical features are used to improve brain tumour detection. Along with the studies done and the implementation of tumour detection systems, the following suggestions can be provided for future researches: By removing areas around the brain (skull), the tumour detection system works more efficiently and it can be applied. The PCNN, in addition to image segmentation, can be used in the pre-processing stage, especially the image filtering.

\section{REFERENCES}

1. Naik, J. \& Pate, S. (2014). Tumor detection and classification using decision tree in brain MRI. IJCSNS, 14(6), 453-468.

2. Shengcong, C., Changxing, D. \& Minfeng, L. (2018). Dual-force convolutional neural networks for accurate brain tumor segmentation. Pattern Recogn., 14, 1-33.

3. Ivana, D., Bart, G. \& Wilfried, P. (2015). MRI segmentation of the human brain: Challenges, methods and applications. Artif. Intell. Med., 10, 1-24.

4. Zexun, Z., Zhongshi, H. \& Yuanyuan, J. (2020). AFPNet: A 3D fully convolutional neural network with atrous-convolution feature pyramid for brain tumor segmentation via MRI images. Neurocomputing., 402, 235-244.

5. Hao, C., Zhiguang, Q., Yi, D., Lan, T. \& Zhen, Q. (2019). Brain tumor segmentation with deep convolutional symmetric neural network. Neurocomputing., 392, 305-313.

6. Sharan, K. (2019). Optimization driven deep convolution neural network for brain tumor classification. Biocybern. Biomed. Eng., 462, $1-15$.

7. Ghassemi, N., Shoeibi, A. \& Rouhani, M. (2020). Deep neural network with generative adversarial networks pre-training for brain tumor classification based on MR images. Biomed. Signal. Process Control, 57, 1-8, https://doi.org/10.1016/j.bspc.2019.101678.

8. Mittal, M., Goyal, L. M., Kaur, S., Kaur, I., Verma, A. \& Hemanth, D. J. (2019). Deep learning-based enhanced tumor segmentation approach for MR brain images. Appl. Soft Comput., 78, 346-354, https://doi.org/10.1016/j.asoc.2019.02.036.

9. Amin, K. A., Moosa, A. \& Foad, K. (2018). Magnetic resonance imagingbased brain tumor grades classification and grading via convolutional neural networks and genetic algorithms. Biocybern. Biomed. Eng., 312, $1-12$. 
10. Khan, H., Shah, P. M., Shah, M. A., ul Islam, S. \& Rodrigues, J. J. (2020). Cascading handcrafted features and convolutional neural network for IoTenabled brain tumor segmentation. Comput. Commun., 153, 196-207, https://doi.org/10.1016/j.comcom.2020.01.013.

11. Eckhorn, R., Reitboeck, H. J., Arndt, M. \& Dicke, P. (1990). Feature linking via synchronization among distributed assemblies: Simulations of results from cat cortex. Neural Computat., 2, 293-307, https://doi.org/10.1162/neco.1990.2.3.293.

12. Rejani, Y. I. A. \& Selvi, S. T. (2009). Early detection of breast cancer using SVM classifier technique. IJCSE, 1(3), 127-130.

13. Syed, A. Q. \& Narayanan, K. (2014). Detection of tumor in MRI images using artificial neural networks. IJAREEIE, 3(9), 36-51, https://doi.org/ 10.15662/ijareeie.2014.0309008.

14. Hall, L. O., Bensaid, A. M., Clarke, L. P., Velthuizen, R. P., Silbiger, M. S. \& Bezdek, J. C. (1992). A comparison of neural network and fuzzy clustering techniques in segmenting magnetic resonance images of the brain. IEEE Trans. Neural Netw., 3(5), 672-682, https://doi.org/ 10.1109/72.159057.

15. Parizel, P. M., van den Hauwe, L., De Belder, F., Van Goethem, J., Venstermans. C., Salgado, R. \& Van Hecke, W. (2010). Magnetic resonanse imaging of brain. In P. Reimer, P. M. Parizel, J. F. M. Meaney \& F. A. Stichnoth (eds.), Clinical MR imaging. Berlin: Springer, 107-195, https://doi.org/10.1007/978-3-540-74504-4_2.

16. Tsai, C., Manjunath, B. S. and Jagadeesan, R. (1995). Automated segmentation of brain MR images. Pattern Recognit., 28(12), 1825-1837, https://doi.org/10.1016/0031-3203(95)00047-X.

17. Fu, J. C., Chen, C. C., Chai, J. W., Wong, S. T. C. \& Li, I. C. (2010). Image segmentation by EM-based adaptive pulse-coupled neural networks in brain magnetic resonance imaging. Comput. Med. Imaging Graph., 34(4), 308-320, https://doi.org/10.1016/j.compmedimag.2009.12.002.

18. Lindblad, T. \& Kinser, J. M. (2013). Image processing using pulsecoupled neural networks. 3rd Ed. Heidelberg, Germany: Springer-Verlag Berlin Heidelberg.

19. Kinser, J. M. \& Johnson, J. L. (1996). Stabilized input with a feedback pulse-coupled neural network. Opt. Eng., 35(8), 2158-2161, https://doi.org/10.1117/1.600797.

20. Kinser, M. J. \& Lindblad T. (1999). Implementation of pulse-coupled neural networks in the CNAPS environment. IEEE Trans. Neural Netw., 10(3), 584-590, https://doi.org/10.1109/72.761715. 
21. Harvard Medical School. (n.d.). Retrieved from http://www.med .harvard.edu/aanlib/home.html.

22. Markell, R. (1994). "Better than Bessel" linear phase filters for data communications. In Linear technology application note 56. Milpitas, CA: Linear Technology Corporation.

23. Gonzalez, R. C. and Woods, R. E. (2008). Digital image processing. 3rd Ed. Upper Saddle River, NJ: Prentice-Hall, Inc.

24. Nobariyan, B. K. \& Daneshvar, S. (2014). A new MRI and PET image fusion algorithm based on pulse-coupled neural network. Paper presented at the 22nd Iranian Conference on Electrical Engineering (ICEE 2014), Tehran, Iran, 20-22 May.

25. Ranganeth, H. S. \& Kuntimad, G. (1995). Pulse-coupled neural networks for image processing. $\mathrm{PhD}$ diss., University of Alabama in Huntsville.

26. El-Dahshan, E. S. A., Mohsen, H. M., Revett, K. \& Salem, A. M. (2014). Computer-aided diagnosis of human brain tumor through MRI: A survey and a new algorithm. Expert Syst. Appl., 41(11), 5526-5545, https://doi.org/10.1016/j.eswa.2014.01.021.

27. Fawcett, T. (2006). An introduction to ROC analysis. Pattern Recognit. Lett., 27(8), 861-874, https://doi.org/10.1016/j.patrec.2005.10.010.

28. Powers, D. M. W. (2015). Evaluation: From precision, recall and F-measure to ROC informedness, markedness and correlation. J. Mach. Learn. Tech., 2(1), 37-63.

29. Dubey, R. B., Hanmandlu, M. \& Gupta, S. K. (2009). Semi-automatic segmentation of MRI brain tumour. ICGST-GVIP Journal, 9(4), 1-8.

30. Wang, H. \& Fei, B. (2009). A modified fuzzy C-means classification method using a multiscale diffusion filtering scheme. Med. Image Anal., 13(2), 193-202, https://doi.org/10.1016/j.media.2008.06.014.

31. Arimura, H., Tokunaga, C., Yamashita, Y. \& Kuwazuru, J. (2012). Magnetic resonance image analysis for brain CAD systems with machine learning. In K. Suzuki (ed.), Medical imaging intelligence and analysis. Hershey, PA: IGI Global, 258-296. http://doi.org/10.4018/978-1-4666 -0059-1.ch013. 